\title{
Sentidos primitivos de la demostración en la formación de profesores*
}

\author{
Primitive sense than demonstration
}

at training for teachers

Artículo Recibido: octubre de 2010

Artículo aprobado: febrero de 2011
Arturo Sanjuán ${ }^{* *}$

Jaime Romero ${ }^{* * *}$

Martha Bonilla****

\section{Resumen}

En este artículo se estudian los primeros sentidos que otorga un grupo de cinco estudiantes para profesor de matemáticas a la actividad de demostrar problemas matemáticos, en el contexto de la continuidad numérica de los números reales. Para dar cuenta de esto, se realizó un experimento de enseñanza, cuya duración fue de un semestre. El análsis de los datos se realizó a través de la elaboración de viñetas.

Se concluye que el grupo otorga tres sentidos primitivos de lo que significa demostrar en matemáticas. Uno de ellos, remeda maneras de la demostración formal y es lo que se propone como demostración paraformal.

\section{Palabras clave}

Aprendizaje de la demostración, demostración que explica, demostración paraformal, intuición, demostración formal.

\section{Abstract}

The first emerging meanings of the proof process, given by a group of five prospective teachers is studied in the context of real numeric continuum. The metodology is a teaching experiment of a six month long. The data analysis is made with de vignettes method.

The conclusion is that there are three primitive meanings of the mathematical proof process. One of them imitates manners of the formal proof and this is what proposed as the paraformal proof.

\section{Keywords}

Proof learning, proof that explain, paraformal proof, intuition, formal proof.

* Artículo derivado de investigación financiada por el Centro de Investiaciones y Desarrollo Científico de la Universidad Distrital.

** Profesor de la Universidad Distrital Francisco José de Caldas, aasanjuanc@udistrital.edu.co

$* * *$ jaimeedumat@udistrital.edu.co

****marthaedumat@udistrital.edu.co 


\section{Introducción}

Cómo se aprende y cómo se puede enseñar a demostrar en matemáticas son preguntas importantes en la comunidad de investigadores de educación matemática en el mundo, debido al fracaso de las formas tradicionales en que se ha propuesto su enseñanza (Duval, 1995, 2004), y a la omnipresencia de la demostración formal para argumentar dentro de la comunidad de matemáticos.

Así, han aparecido enfoques alternativos para abordar la enseñanza y el aprendizaje de la demostración matemática. Esta investigación tiene en cuenta tres de ellos: el constructivista, el cognitivosemiótico y el sociocultural.

Del enfoque constructivista se consideran los trabajos de Balacheff (1987), Hanna (1990) y Simon (1996). Este último, al igual que el aquí presentado, fue realizado en formación de profesores.

Hanna (1990) tipifica la demostración en dos clases: la de las demostraciones que explican y la de las demostraciones que demuestran. En las demostraciones que explican, las personas usan representaciones, ejemplos y formas discursivas propias que les permiten argumentar sus producciones. Las demostraciones que demuestran son aquéllas que cumplen los requisitos pretedidos por la comunidad de matemáticos. Es decir, demostraciones formales, ancladas en teorías dadas por axiomas (Goldsern \& Judah, 1998).

Para analizar la demostración que explica, Balacheff (1987) introduce, según la formalidad de la deducción alcanzada, unos estadios o niveles jerárquicos de argumentación, a saber: la experimentación intuitiva que consiste en argumentar una afirmación a través de uno o varios ejemplos; la experimentación crucial que consiste en buscar algún ejemplo que ponga a prueba la afirmación; la experimentación genérica que consiste en argumentar de manera general haciendo uso de un ejemplo; $y$, por último, la experimentación mental que consiste en argumentar de manera deductiva desprendida de los ejemplos.
En esta misma dirección Simon (1996) introduce dos nuevos niveles; en el nivel 0 quedan los enunciados que no son argumentos, y, en el nivel 1, quedan los pronunciamientos que argumentan basados en la autoridad. Los niveles 2, 3, 4 y 5 corresponden a los experimentos intuitivo, crucial, genérico y mental de Balacheff (1989), respectivamente.

El enfoque constructivista adopta una posturalakatosiana, a propósito de la organización epistemológica de la demostración en matemáticas como objeto de la educación en esta disciplina. Este organizador, al aceptar el principio de falibilidad de la ciencia (Popper, 1959), operativizado en matemáticas mediante la dinámica prueba/refutación, (Lakatos, 1976), organiza la experimentación y relativiza la garantía de la verdad de las proposiciones matemáticas.

Por su parte, en el enfoque cognitivo semiótico sobresalen los trabajos de Tall (1989) y de Duval (1995). Para este autor, la cognición en matemáticas supone un proceso semiótico de conversión de registros de representación, que implica discriminar unidades significantes de los distintos registros y poner en coordinación las unidades de uno con las del otro. Duval (2004) ha elaborado una propuesta de enseñanza de la demostración soportada en su teoría cognitiva.

Duval llama exploración libre a la demostración que explica de los constructivistas, adjudicándole alguna importancia para aprender la demostración que demuestra (Hanna, 1990), sin dedicar muchas líneas al tema; para él el sentido de la demostración es el de la prueba formal.

La teoría de las representaciones semióticas produjo clasificaciones de las proposiciones, según el tipo de sentido involucrado, cuando se las examina en la trama de la demostración formal; que además son útiles para esta investigación. Tal teoría incorpora la toma de conciencia de esos tipos de sentidos en la toma de conciencia de la demostración.

En primer lugar, es necesario que los estudiantes entiendan el significado de las proposiciones en 
tanto su relación con la enunciación. Este significado tiene, al menos, dos dimensiones: la dimensión lógica, relativa al valor de verdad y la dimensión epistémica, relativa al conocimiento y la necesidad de la proposición.

En segundo lugar, es necesaria la toma de conciencia de relaciones operatorias (de carácter discursivo) y teóricas (de carácter disciplinar) de las proposiciones con otras proposiciones. Éstas tienen, al menos, tres estatus: el estatus operativo, en relación con otras proposiciones del teorema como la hipótesis o la tesis; el estatus teórico, en relación con otras proposiciones de la teoría como los axiomas y otros teoremas, y, el estatus normativo, relativo a las creencias.

Del enfoque sociocultural, por una parte, de los trabajos sobre demostración, se toma aquí el de Hemmi (2008), fundamentado en las comunidades de práctica. En éste, Hemmi concluyó que el aprendizaje de la demostración se da en la práctica de demostrar, y que es deseable tematizar explícita e implícitamente qué es una demostración.

Por otra parte, del enfoque sociocultural se toman los indicadores principales de aprendizaje y sentido. Específicamente, de la Teoría cultural de la objetivación (Radford, 2006) y de las comunidades de aprendizaje (Llinares \& Oliveros 2008), éstas, a su vez, se soportan en la Teoría de las comunidades de práctica (Wenger, 2001).

Aunque los enfoques señalados parecen contradictorios entre sí y con los aspectos sustentados en las comunidades de práctica, el equipo investigador, en consonancia con Llinares (2006); D'Amore (2006) los asume más bien como complementarios.

\section{Problemática y pregunta a abordar}

En los programas de formación de profesores de matemáticas, una preocupación recae sobre comprender los procesos mediante los cuales los EPP (estudiantes para profesor) aprenden a demostrar, que particularizada al contexto en el cual se desarrolló ésta investigación, implica comprender estos procesos cuando los EPP participan en experimentos deenseñanza que asumen la resolución de problemas de demostración matemática como ambiente de aprendizaje.

En relación a la comprensión de los procesos de aprender a demostrar es importante ubicar sentidos que los estudiantes adjudican a la demostración matemática. En este artículo se da cuenta de algunos sentidos de la demostración que explica y un sentido que aparece cuando los estudiantes se plantean realizar la demostración formal y abandonan la demostración que explica.

En síntesis, asumiendo una perspectiva teórica que considera como complementarios los enfoques constructivistas, cognitivo semiótico y sociocultural, en este artículo se analizan sentidos que los EPP partícipes del curso Problemas del Continuo, elaboraron en el proceso de aprendizaje de la demostración matemática.

\section{Metodología}

El experimento de enseñanza se desarrolló en el curso en el que participaron 35 estudiantes, denominado, Problemas del continuo, asignatura del programa de formación de profesores de matemáticas de la Universidad Distrital Francisco José de Caldas, que problematiza el continuo numérico y se desarrolla a través de la resolución de problemas.

\section{Población}

Para el análisis se seleccionó un grupo de cinco estudiantes, cuatro mujeres (Ángela, A; Katherine, K; Loli, L y Cindy, C) y un hombre (Johnathan, J). Estos estudiantes han tenido experiencias tanto en resolución de problemas, como en clases tradicionales; en ellas han tematizado aspectos de la geometría euclidiana, los sistemas de numeración y el pensamiento multiplicativo.

\section{Experimento de enseñanza}

La metodología propuesta para el desarrollo del presente proyecto se apoya en los postulados de la investigación cualitativa interpretativa, particularmente en la metodología denominada Experimento de Enseñanza. De la literatura en investigación 
en educación matemática se destacan los trabajos de Cobb, Confrey, Disessa, Lehrer y Schauble (2003), Steffe y Thompson (2000), quienes han utilizado los experimentos de enseñanza para dar cuenta de los aprendizajes de los estudiantes. Callejo, et al. (2007), citando a Gravemeijer, comenta que este tipo de investigación "difiere de la investigación acción participativa en tanto el objetivo no es ofrecer una secuencia instruccional que «funcione», sino una teoría empíricamente fundamentada sobre cómo piensan los investigadores que un cierto conjunto de actividades instruccionales podrían funcionar (Gravemeijer, 2004:111)”.

Para los autores mencionados, un experimento de enseñanza se estructura en tres fases: planeación y diseño, experimentación y análisis retrospectivo. Las fases para este experimento se detallan a continuación.

En la fase de planificación y diseño el equipo de investigadores, incluyendo al profesor, eligió la situación problema a presentar a los estudiantes, y definió como ambiente de aprendizaje la resolución de problemas (Santos-Trigo, 2007; Mason, Burton \& Stacey, 1989; Sanjuán \& Romero, 2009; Sanjuan \& Bohórquez, 2008) entendida como una empresa de las comunidades de aprendizaje emergentes en el aula. El aprendizaje se concibe como producto de la constitución y participación en la empresa, logrando un proceso paulatino de tomas de conciencia, llamado "objetivación en la teoría cultural de la objetivación” (Radford, 2006); pero, también como uso, transformación y elaboración de modelos mentales (D'Amore, 2006a) e intuitivos (Fischbein, 1999).

Como hipótesis de ruta de enseñanza el grupo de investigadores asumió la participación de los estudiantes en ciclos (Figura 1) de resolución de problemas de demostración (Sanjuán, Romero \& Bonilla, 2008); (Bohorquez, Romero \& Bonilla, 2009), segmentados en momentos caracterizables por la dirección de la toma de conciencia lograda; es decir, por la ladera de generalidad, declarada por los estudiantes, de la demostración como objeto de la educación matemática.
Como hipótesis para la ruta de aprendizaje el grupo de investigadores asumió, por una parte, la existencia de una relativa continuidad entre los distintos momentos que dan forma a la demostración que explica, empezando por los momentos dedicados a la exploración del objeto $\mathrm{A}$, más adelante definido, y a la actividad de conjeturación, seguidos por los momentos centrados en las elaboraciones de los distintos experimentos intuitivo, crucial, genérico y mental; el entrelazamiento de estos momentos contituye un ciclo de resolución y permitirá la toma de conciencia de este tipo de demostración; por otra parte, asumió la existencia de una ruptura relativa entre la objetivación de la demostración que explica y la objetivación de la demostración que demuestra, haciendo necesario que la correduría (como actividad del profesor) involucre instrumentos provenientes de la teoría de las representaciones semióticas para provocar la transición.

\section{Fase de experimentación}

Como punto de partida de la ruta de enseñanza el profesor formuló el enunciado siguiente: «A es el conjunto de los números del intervalo $[0,1]$, en cuya expansión decimal solo hay ceros, cincos o nueves.», solicitando de los estudiantes trabajar en grupos colaborativos, tener en cuenta el conjunto $A$ para plantearse preguntas, conjeturas y un problema de demostración que debían resolver.

Para resolver la situación problema planteada por cada uno de los grupos de estudiantes, se contaba con las 32 sesiones de dos horas cada una, que comprenden el tiempo asignado institucionalmente a un curso semestral. La participación de los estudiantes en los diferentes ciclos de resolución de problemas se dió en grupos colaborativos y en sesiones de gran grupo.

La organización de la clase supuso el trabajo en grupos colaborativos constituidos libremente (de 4 a 5 personas). Cada grupo se dió un nombre y trabajó el problema que él mismo se planteó. Se trabajó en 2 sesiones semanales de 2 horas cada una, durante las cuales los EPP trabajaron en un salón de clase convencional. Durante el semestre los EPP 
acudieron, cuando lo consideraron conveniente, a consultar libros, Internet, otros profesores, compañeros del proyecto curricular o al uso de software especializado.

La intervención del profesor en cada grupo colaborativo se dió por solicitud de los grupos o del profesor; trataba de ayudarles a fijar, establecer y comprender distintos puntos de vista propuestos por los miembros del grupo, con la intención de favorecer e instrumentar la constitución de comunidades de aprendizaje. Por ello siempre utilizó, como aproximación, el formato de pregunta para provocar la participación de los miembros llevándolos a usar, elucidar y transformar sus modelos mentales e intuitivos. En las sesiones de gran grupo el profesor intervino como representante de la comunidad de la comunidad de profesores de matemáticas, $y$, como corredor entre los distintos grupos colaborativos de la clase.

\section{Fase de análisis retrospectivo}

El grupo de investigadores observó y analizó el desarrollo de la experimentación, discutiendo los escritos de los estudiantes, las intervenciones en pequeño grupo y las exposiciones en gran grupo, apoyándose en la teoría que fundamentaba la hipótesis de aprendizaje y en la experticia de los investigadores. De este proceso continuo resultó el redireccionamiento de la acción de la clase en los casos en que se consideró necesario.

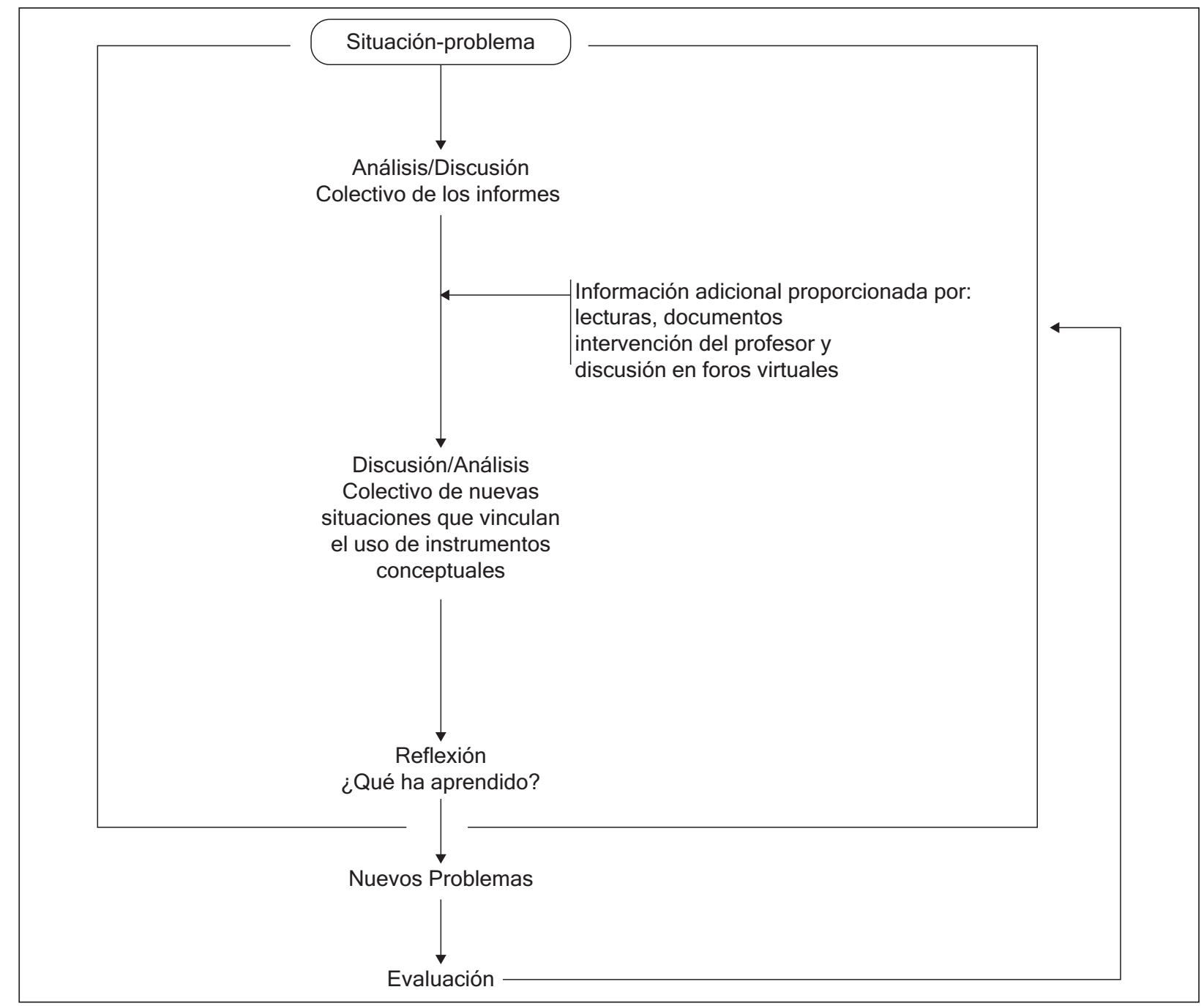

Figura 1. 
Esta fase se centró en indagar la plausibilidad de la hipótesis de ruta de aprendizaje en relación con la actividad (cognitiva y social) desplegada y si el diseño de los ciclos de resolución de problemas, incluida la situación problema planteada, generaba en los EPP la actividad matemática esperada, obteniendo reformulaciones teóricas que incidieron en el diseño actual y que proveen explicaciones acerca del aprendizaje logrado.

\section{Análisis de los datos}

El análisis de los datos se inscribe en la perspectiva cualitativa, tomando como unidades de análisis segmentos de la clase de Problemas del Continuo. Estos segmentos de clase fueron obtenidos tomando como criterio la emergencia o la evolución de alguno de los sentidos acerca de la demostración en las configuraciones de los EEP.

El proceso de análisis se realizó en dos fases secuenciales, la primera de carácter descriptivo y la segunda de carácter inferencial (Gavilán, García \& Llinares, 2007). Este proceso tuvo como objetivo analizar el contenido de los escritos, videograbaciones y audios que dan cuenta de la actividad matemática de los estudiantes, con el propósito de identificar los sentidos dados a la demostración matemática.

Como resultado del proceso de análisis se construyeron las "viñetas". La viñeta, caracterizada como un instrumento de investigación complejo, permite a los investigadores integrar información procedente de las diferentes fuentes, actores y relaciones entre ellos lo que, para el caso de esta investigación, hace viable la integración de los enfoques constructivista, cognitivo-semiótico y sociocultural adoptados. Desde el punto de vista conceptual, el término viñeta es utilizado en el sentido dado por Gavilán, et al. (2007), adecuándolo a la práctica de resolución de problemas de demostración realizada por los EPP.

La viñeta es un informe sobre aspectos de la práctica [...] que integra información de diferentes fuentes, transcripciones de las sesiones de clase, los informes elaborados en el análisis descriptivo, [el ciclo de resolución del problema de demostración], y la descripción e interpretación de lo que sucede en los segmentos [del ciclo de resolución de problemas]. En las «viñetas» además se integran inferencias realizadas por los investigadores para mostrar qué interpretaciones se han realizado y su vinculación con la evidencia empírica (p. 161).

Se elaboraron dos viñetas, cada una de las cuales está estructurada de tal manera que su desarrollo permite una contextualización de los datos analizados, acompañada de segmentos que reconstruyen sucesos de clase. Asimismo, incluyen descripciones y análisis de los datos, las inferencias elaboradas por los investigadores y sus argumentaciones acerca de la plausibilidad del conjunto de interpretaciones realizadas.

Para el análisis sobre los sentidos que los estudiantes dan a la demostración matemática, el grupo investigador utilizó categorías definidas en el marco teórico e incorporó, para la demostración que explica, la categoría denominada demostración paraformal, dando cuenta de un tipo de demostración no incluida en la teoría de la demostración que explica. La demostración paraformal se caracteriza porque en ella se pueden identificar ausencias en la dimensión epistémica o lógica con relación a las proposiciones que conforman la trama argumental. Es decir, en dicha trama existen ausencias del cambio en el valor epistémico o de transferencia local del valor de verdad o de control del estatus teórico.

Los segmentos de clase, que fueron analizados de manera simultánea, corresponden a la resolución de los siguientes tres problemas de demostración, planteados por los EPP: 1) demostrar que es el mínimo del conjunto $A, 2$ ) demostrar que es el máximo del conjunto $A$ y 3 ) es igual a 1 .

\section{Resultados}

Los resultados serán expuestos a continuación en dos viñetas Gavilán, et al. (2007: 161). En la primera se exponen los sentidos de la demostración que explica (Hanna, 1990) ligada a los ejemplos y el uso que dan los estudiantes a los mismos. En la segunda, la aparición y reaparición de la demos- 
tración paraformal en las producciones de los estudiantes.

Los estudiantes vivencian una práctica de la demostración que explica

Los datos de esta viñeta son tomados de la segunda (11 de agosto de 2010) y de la octava sesión (3 de septiembre de 2009). Los EPP están haciendo sus primeras aproximaciones a la situación, se les ha pedido que generen preguntas sobre el conjunto $A$ para elaborar una conjetura. Las primeras experimentaciones de los estudiantes van dirigidas a familiarizarse con el enunciado de la situación.

A: Sea A.

$\mathrm{K}$ : Bueno, se entiende un conjunto entre 0 y 1. $[\ldots]$

A: Pero luego no decía, solo ceros, solo cincos, o solo nueves.

K: Pero tú misma dijiste, que pueden estar los tres.

L: Ah, o sea, no cabe un 0,3.

A: No, sería 0,05 o 0,059 ¿sí?, ¿pero seguidos?, ¿podría ser?, o 0,59.

Transcripción 1. Familiarización con el enunciado

Al tiempo que se familiarizan con el enunciado, los estudiantes se dan cuenta que los elementos del conjunto son números decimales en el intervalo [0, 1] (ln. 1, tr. 1), asumen que en la expansión decimal debe haber solo ceros, cincos o nueves pero que pueden estar combinados uno, dos o tres de estos dígitos y estar o no repetidos (ln. 3-6).

La evidencia (tr. 1) muestra que el grupo de estudiantes está familiarizándose con un objeto matemático a través de ejemplos de características de dicho objeto. Es decir; a través de particularizaciones, hecho usual en la actividad matemática general.

En el fragmento de discusión que sigue los estudiantes se están poniendo de acuerdo acerca de las preguntas que deben elaborar; la discusión los lleva a tratar de entender los elementos y la estructura del conjunto.
A: [...] ¿es que no es como más sencillo mirar lo de secuencias?, o sea digamos cero, no sé qué, entonces en qué momento se va a volver a repetir.

J: ¿Es que sabemos que es infinita la expansión?, ¿cierto?, pero... entonces ¿cómo podemos relacionar lo infinito con lo decimal?

K: Teniendo en cuenta...

$J$ :...teniendo en cuenta el cero, el cinco o nueve. ¿Y ahí cómo se ve eso?

K: Por qué es infinito.

J: Por qué es que digamos...

K: Luego digamos que es, 0,59. El anterior a ese ¿cuál sería?... ¿No se podría poner 0,8 ?

C: 0,50 .

L: ¿0,50? ¿Y por qué no 0,55?... ¿Y después del 0,50?

$K:$ ¿Después, o antes?

L: Antes, sí.

A: 0,05 .

$L: ¿ 0,9 ?[\ldots]$

A: Si: 0,9 .

L: Si ve, ni siquiera sabemos. ¡Qué complique!

C: ¿Qué?

K: Que ella estaba haciendo una secuencia, entonces está preguntando que antes del 0,50 qué número va.

A: Antes sería, pues lo del 0,50, ¿Qué va?

J: Cuya expansión decimal...

A: Por eso, cero, 0,09 y entonces va...

L: ...o 0,9 .

A: 0,9 .

C: ¿Antes?, no, ese sería después.

$K$ : Ese sería después.

A: Ese sería después.

L: Ah sí, claro.

A: Ya, ya, ya sería 0,9 porque estamos hablando de que...Segunda

C: O sea 0,009. 
L: O sea, el primero es cero coma ...

A: 0,$00 ; 0,00$

L: No porque...

A: Ya.

C: Pero teniendo en cuenta que tiene infinitos decimales, por qué si el anterior a éste, digamos éste 0,59 podría ser 0,559 y después 0,005.

K: O sea que solo estamos tomando solamente dos decimales después del 0.

L: Mejor dicho, después del cero ¿qué sigue?,

A: 0,05 .

K: Por qué 0,0.

L: 0,005

K: Sí.

L: O mejor el 0,0005.

Transcripción 2. Experimentación sistemática

En la línea 1 (tr. 2), Ángela muestra una intención de preguntar algo relacionado con secuencias, porque lo considera más sencillo y, en la línea 2, Johnatan pregunta por la relación entre "lo infinito" y "lo decimal". Estas dos intenciones encaminan la experimentación. Así, con los ejemplos que en el diálogo van construyendo colectivamente (ln. 5-40) están tratando de mostrar infinitos elementos ordenados.

En la experimentación tratan de ejemplificar la estructura de orden; en el diálogo entre Cindy (ln. 33) y Katherine (ln. 34) ponen en claro que, debido a que la expansión decimal es infinita, es necesario tomar ejemplos con más de dos dígitos. La interacción dialógica entre Loli, Ángela y Katherine (ln. 35-40), que desarrolla la precisión obtenida en las líneas anteriores, va cargada de una técnica que les permitirá encontrar cada vez, números del conjunto entre un número dado del conjunto y cero.

En el propósito de formar una sucesión, al parecer siguiendo la insinuación de Ángela, los estudiantes dirigen su experimentación a encontrar sucesores y antecesores de elementos, todos ellos dentro del conjunto. Ligado al uso de la técnica encontrada, emerge como manera de refutar, el contraejemplo (ln. 7-9, 35-40, tr. 2).

Las experimentaciones guiadas por las intenciones, entender el conjunto, tener los elementos, hacer una secuencia, construir lo decimal, o infinito, pueden ser entendidas como ciclos colectivos y entrelazados de atascamiento/ajá (Mason, et al., 1989), en el sentido de que cada vez que van comprendiendo una pregunta que intermedia su intención -por ejemplo construir la secuencia, está intermediada por saber cuál es el primer elemento del conjunto- producen una comprensión que es asumidad por todos y que orienta su exploración siguiente.

Vemos emerger mediante una contrucción colectiva que responde a una experimentación, dirigida de manera relativamente inconciente, por dos insinuaciones iniciales y desarrollada a lo largo de sucesivos ciclos colectivos atascamiento/ajá, que conduce a Loli (ln. 29) a preguntar para los otros y para sí misma sobre el mínimo de $A$ : O sea, el primero es cero coma... De esta manera emerge una técnica que permite generar una secuencia infinita totalmente ordenada de elementos de $A$ con menor elemento, que a su vez contesta a la insinuación de Loli.

Luego de establecer características del conjunto $A$, ligadas a secuencias, orden y densidad, los estudiantes dirigen su atención a la construcción de una pregunta que guiará sus siguientes experimentaciones, para ello buscan la ayuda del profesor y le solicitan que les indique si la manera que estaban usando para establecer el orden entre dos números era o no correcta. En el siguiente fragmento de clase se muestra cómo la dirección del diálogo entre el profesor y el grupo los llevó a elaborar una pregunta, una conjetura y a tomar conciencia que ahora la actividad del grupo se dirige a demostrar la conjetura.

$$
\begin{aligned}
& \text { L: ¿será que el 0,999999... es el último? } \\
& \text { P: Por ejemplo. } \\
& \text { J: Loli ¿cómo? } \\
& \text { P: ¿Cuál es el más grande? }
\end{aligned}
$$


A: ¿Cuál es el más grande?

$P$ : ¿Cuál es el más grande del conjunto? Ahí ya tienen la pregunta...

L: ¡Voy a escribir ajá bien grande!

[el profesor se retira]

J: ¡Eureka! o sea que ¿quién, cuál es el más grande? Sí, 0,999999 periódico.

A: 0,99 periódico es el más grande.

L: ¿Será?

J: ¿Cómo?

K: Y así mismo se puede sacar el más pequeño.

L: Sí.

A: Bueno, pero eso hay que demostrarlo, ¿pero cómo lo demostramos, que es el más grande?, ¡esa es la pregunta!

K: ¡Esa es la idea!, ¡esa es la idea!

Transcripción 3. Surgimiento de la conjetura

El trabajo de ejemplificación previo y las intervenciones del profesor (ln 2,4 tr. 3) los lleva a decir que es el más grande del conjunto. En $(\ln 15,16)$, el grupo de estudiantes instala una conjetura y toman conciencia de que ahora la cuestión es cómo demostrarla.

La siguiente transcripción (tr. 4) es una continuación de la anterior (tr. 3). Loli está tratando de demostrar que es el más grande del conjunto.

L: ¡Contraejemplo!, porque no va a haber otro, porque en cualquier momento va a haber un cinco. Sí, o sea, supongamos $0,9999999 \ldots 5$ y si yo digo que 0,9999999 siempre nueve...

A: Que eso fue lo que dijimos con el profe, que igual si comenzamos a comparar con el nueve entonces no importa que siga después.

L: Justo en ese preciso momento cuando llegue al cinco, nueve va a ser más grande que el cinco y va a seguir siendo el 0,99999... más grande,

K: Más grande,

A: Más grande,

L: más grande que todo...
A: ...jentonces qué?, ¿ponemos ese ejemplo?, ¿el de el 0,99995 y 0,9 periódico?, pero es que igual al tener 0,9 periódico ya estamos diciendo que igual va a ser el más grande.

Transcripción 4. Experimentación crucial y genérica como argumentación

En la línea 1 (tr. 4), Loli expone un ejemplo que llamó, de manera incorrecta, contraejemplo. Es interpretado como un ejemplo que intenta poner a prueba la conjetura del grupo; es decir, se trata de un experimento crucial (Balachef, 1987). En la línea 3, Loli usa este mismo experimento para argumentar, de manera general, por qué él es el más grande del conjunto. Los estudiantes están produciendo formas discursivas propias útiles para argumentar; es decir, han hecho una demostración que explica (Hanna, 1990); ahora bien, en tanto explicación general a través de un ejemplo, se trata de una demostración que explica dentro del estadio experimento genérico (Balachef, 1987) o de una argumentación general atada al ejemplo (Simon, 1996).

El análisis de los datos, hasta este punto, permite ver que la experimentación fue usada para familiarizarse con el objeto matemático ligándolo a secuencias, orden y densidad; al parecer, intentando ordenar el conjunto totalmente y de manera discreta como una sucesión infinita, asunto que es imposible matemáticamente. Esto permitió a los estudiantes tener una aprehensión del conjunto y -por ende- de la situación (formular conjeturas). Así, los experimentos o ejemplos que realizaron los estudiantes fueron dirigidos, en últimas, a tener una aprehensión de la situación que parece integrar las perspectivas de Ángela (la situación como un asunto relacionado con sucesiones) y Johnatan (la situación como un asunto que relaciona lo decimal y lo infinito). Esta estrategia de aprehensión de la situación, con un conjunto similar y con otros estudiantes, se reporta en Sanjuán y Romero (2009).

Los estudios realizados anteriormente sobre demostración, Balachef (1987), Hanna (1990) y Simon (1996), concuerdan con este estudio en que la experimentación y la argumentación van de la mano. Los estudiantes usaron los ejemplos y los contrajemplos, estimulados por sus preguntas y las 
del profesor, manteniendo un sentido de su actividad matemática argumentativa regulada sistemática y permanente, al estar orientados por sus propias intenciones o perspectivas.

El hecho de no exponer una situación con la petición de "encuentre que" o "demuestre que" y, a cambio, exhibir una situación sobre la que los estudiantes deben inquirir; conlleva a que la experimentación sea usada para producir preguntas y conjeturas, desarrolladas en ciclos atascamiento/ ajá, y que la conjetura principal sea un producto de conjeturaciones y refutaciones más pequeñas. La actividad desarrollada en el pequeño grupo colaborativo va introduciendo a los EPP en las tareas propias de una comunidad matemática guiada por un sentido quasi-empirista de las matemáticas (Lakatos, 1976) -así la conformada por ellos sea una microcomunidad.

\section{La ruptura de la práctica regulada de la demostración que explica, por la práctica normativa de la demostración paraformal}

Los datos de esta viñeta corresponden a la tercera (13 de agosto de 2009), cuarta (18 de agosto de 2009) y séptima sesión (27 de agosto de 2009) y provienen de las videograbaciones de la clase y de los cuadernos del resolutor de Katherine, Ángela y Cindy. El siguiente fragmento corresponde a la tercera sesión. El grupo de estudiantes es conciente de que la tarea consiste ahora en demostrar que es el más grande del conjunto. Están discutiendo de qué manera podrían hacer esto.

L: Entonces tenemos que demostrarlo, entonces partamos de que vamos a demostrar que 0,99 periódico es el más grande.

C: Pero para eso, tendríamos que buscar...

L: ...teoría.

C: ¡Sí!

K: ¡Claro!

A: Pero, jobviamente!

L: Pero, ¿qué libro?

A: Pero, ¿qué libro?
C: Pues uno sobre ordinalidad, pero ¿en dónde?

[...]

L: Entonces buscamos un libro

A: Entonces buscamos un referente teórico, ¿sí o no?

L: Un libro para justificar.

A: Para poder demostrar en general que 0,9 periódico es el mayor del conjunto.

J: Cuál es el más grande y cuál es el más pequeño. Ahí estamos mirando.

A: Eso es de cardinalidad y de orden, pero es que estamos buscando todo eso de orden, porque el profesor dijo que era de orden, porque él había buscado que era orden.

$[\ldots]$

C: ¿Sí encontraste algo ahí?

K: Por eso, eso es lo que está diciendo Ángela.

A: Que debemos buscar la relación, partiendo de que es una relación de orden.

J: Es que aquí podemos ver que es una relación de orden, pero partiendo de una relación binaria.

$[\ldots]$

A: Entonces deberíamos buscar eso, y buscar lo que dice el libro, lo que es respecto a una relación, ¿listo?

Transcripción 5. Las demostraciones, las teorías y los libros

En el grupo aparecen distintas ideas sobre la utilidad del libro para demostrar que es el más grande del conjunto: encontrar referentes teóricos, justificar, demostrar en general y hallar temas específicos, así, los estudiantes tratan de hallar qué es una relación de orden (ln. 15-20), pero no se preguntan para qué sirve este objeto matemático en su tarea de demostración. Aparece la idea de que en los libros se encuentran las razones que permitirían configurar la demostración de manera general (ln. 10-13).

$\mathrm{Al}$ inicio de la sesión 18 de agosto de 2009 el grupo comenta las ideas que han tenido acerca de cómo demostrar que la conjetura es el más grande del conjunto. Reiteradamente, y en un lapso de cinco minutos, Katherine y Ángela presentan cuatro ve- 
ces las dos ideas siguientes: comenta que ha intentado tener en cuenta «lo de la relación de orden» y ha hecho una afirmación pero que entró en un ¡atasco! [luego de investigar acerca de la relación de orden y seriación, y las otras definiciones necesarias, ¡¿qué se debe hacer con el problema?!] Ángela responde que «es eso [...] la idea es cómo llegar a una generalización [...] que es mayor entre todos los de 0,$9 ; 0,99 ; 0,9$ no sé qué (mientras pronuncia cada número pone la mano izquierda como referencia fija y aleja de ella su antebrazo derecho hacia la derecha; en cada pronunciamiento el antebrazo derecho se aleja más que en el caso anterior; en el último, lo desliza y extiende la mano hasta quedar señalando el horizonte de su lado derecho) y ahí demostramos que es antisimétrico porque no es igual a ninguno de los otros».

Durante este diálogo Loli ha manifestado no saber cómo hacer la demostración, y que «lo primero es demostrar que este número [refiriéndose a ] existe en el intervalo; de pronto, se decide a presentar un escrito (Figura 2) que ha elaborado en correspondencia con los acuerdos a los que llegó el grupo en la sesión anterior.

Loli comparte para la discusión el escrito en el que ella cree haber demostrado de manera general «que cero nueve es más chiquito que cero nueve, nueve....».

Figura 2. Demostración paraformal

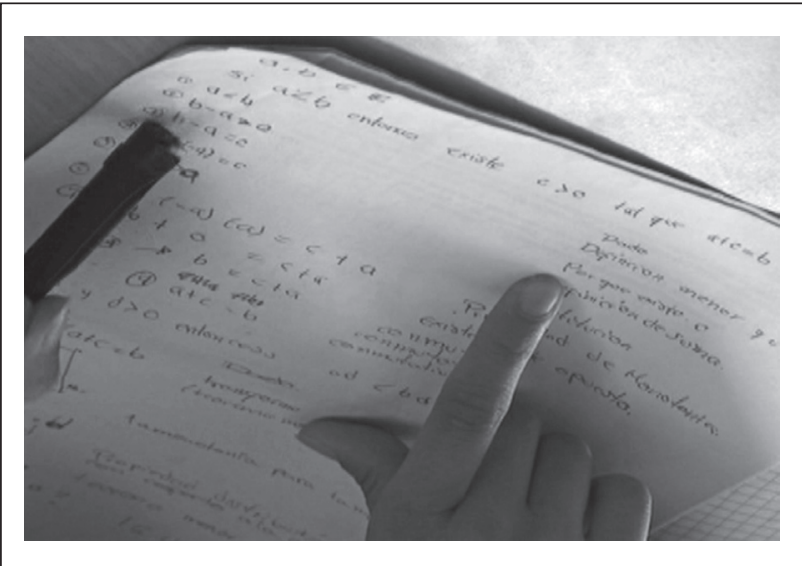

En el resto de esta sesión las estudiantes dirigieron sus esfuerzos a examinar el escrito de Loli. En la discusión sostenida (apartes de la cual se presentan en la transcripción 6) logran dar sentido al texto, en tanto demostración general, y a cada una de las proposiciones que lo constituyen; además, muestran tener control de la validez de cada uno de los pasos.

$\mathrm{K}:[\ldots]$ si a es menor que b existe un c... Bueno, hasta aquí íbamos bien, bueno de aquí abajo no entendí, bueno, mira, dice que a y b, digo que a es mayor [se equivoca y luego corrige diciendo menor que] que b, que eso es lo dado

$K:[. .$.$] Entonces b$ - a es mayor que cero, por la definición de menor que. $b$-a es igual a c porque existe $c$, $y$ pues se sabe que $c$ [existe]. b más menos a es igual a c. [indicando la expresión $b+(-a)=c$ ]

A: Pero bueno es para dejarlo positivo, porque mira más por menos, menos, o sea tengo lo mismo que arriba [señalando que la expresión $\mathrm{b}+(-\mathrm{a})=\mathrm{c}$ es equivalente $\mathrm{a} \mathrm{b}-\mathrm{a}=\mathrm{c}]$

K: Ah bueno, ¿c es mayor que cero o que a?

Que cero

A: Pero bueno esto ya estaba acá. [indica en el escrito de Loli la expresión $c>0$ ]

C: Pero bueno ¿esto no obligaría a que a y b fueran iguales?

L: no, tiene que ser uno más grande que el otro... pues según yo.

C: No, tiene que ser igual porque se le está sumando $y$ restando $a y b$.

\section{A no, espérate.}

A: Pero ahí sí se podría estar diciendo que son desiguales porque se está sumando.

C: si es suma sí, pero si es restando, no puede ser.

A: ¿Por qué?...

C: porque ¿Cómo le va a estar restando a un número igual otro diferente, que dé cero?

A: Sí, es verdad.

L: Pero dió un c...

[risas]

[...] 
L: Pero ahí no dice que es igual a cero

A: No, ¿ahí qué dice?

L: Lo que dice es que c es mayor que cero, o sea que ninguno de esos dos, que éste menos éste debe dar algo mayor que cero.

A: Necesariamente...

$[\ldots]$

Pero, ¿entonces aquí...aquí no habría un signo? [señala sobre (-a) (a) en el sexto renglón del texto de Loli].

L: No, porque a cosas iguales se le suman cosas iguales, las cosas...

$k:$ ¡Por eso!

A: Las cosas son iguales.

L: Mira es que a éste se le puso éste [señala la expresión del renglón 6].

A: Sí, sí estoy de acuerdo.

L: Entonces no se ha desequilibrado digamos..., la ecuación. Entonces digamos esto es cero, menos y menos da más, entonces $b$ más 0 es igual a un c...más un $a$.

K: Sí.

L: Pues ahí está, ¿no? que b es igual...

A: a c más a.

[...]

A: [...] para eso se hizo todo el procedimiento, porque al decir que $b$ es igual a a un ¿qué? a un... c menos $b$, [se confunde con $b$-c] pues estamos diciendo que b va a ser mayor ¿si me hago entender? Sí porque le estamos colocando otra cosa ahí al lado para que sean iguales.

K: Ujum.

A: Pues yo creo que sí, eso sí se puede hacer así, pues por lo que se hizo todo el procedimiento.

K: Listo, entonces tocaría hacer esta demostración pero con los númeritos que vamos a utilizar ¿cierto?

A: Hay que hacer la demostración pero con 0,9 y 0,9 periódico,...

K: ¡Por eso!

$[\ldots]$
C: ¿Pero antes de todo eso [señala el texto que trajo Loli], no tocaría demostrar las propiedades? [se refiere a las propiedades de orden].

$[\ldots]$

A: Pero es que con este coso [señala el texto de Loli] estamos demostrando que es anti simétrica, ¿no era? Para demostrar que el uno no es igual al otro.

$[\ldots]$

A: $\mathrm{Si}$ a es menor que $\mathrm{b}$ entonces existe un $\mathrm{c}$ menor que cero tal que $\mathrm{a}+\mathrm{c}=\mathrm{b}$. Entonces coloquemos Afirmación-Razón... ... pues al demostrar eso entonces ya tenemos que sería antisimétrico. ¿Si? Entonces nos tocaría demostrar las otras dos propiedades, ¿cuáles eran?

Transcripción 6. Aparece por primera vez la demostración paraformal

En el diálogo inicial, se manifiesta que el grupo se está poniendo de acuerdo acerca de cómo demostrar la conjetura. Katherine quiere vincular lo que ha averiguado acerca de relaciones de orden y seriación con el problema pero no sabe cómo hacerlo. Ángela, quiere demostrar que $\mathrm{a}<\mathrm{b}$ es antisimétrica; piensa que si se demuestra que es más grande que todos los números de expresión decimal finita y conformada solo con 9 s, se demuestra que no es igual a ninguno de esos otros números $\mathrm{y}$ entonces la relación $\mathrm{a}<\mathrm{b}$ es antisimétrica.

Pasando a la discusión del escrito de Loli, en ella se revela que:

1. mientras que Loli y Katherine lo perciben como una demostración general de que si $\mathrm{a}<\mathrm{b}$ entonces $\mathrm{b}>\mathrm{a},($ tr. $6 \ln$. 28-30). Ángela, aunque acepta la percepción de Loli y Katherine, ve en lo que ellas perciben un paso necesario en la demostración de que $\mathrm{a}<\mathrm{b}$ es antisimétrica, (tr. $6 \ln .11,30,31,39)$.

2. después de aceptar el texto de Loli como una demostración, el grupo manifiesta que es necesario hacer la demostración pero con y con todos los números de expresión decimal finita y conformada solo con 9s. (tr. $6 \ln$. 35-38). 
3. La percepción de Ángela fue tácitamente aceptada, como se puede ver en sus cuadernos del resolutor, dado que la palabra antisimetría encabeza los textos, resultado de la discusión y lo expresado en (tr. 6 ln. 39). Pero, a cambio, las EPP pensaron dirigir sus esfuerzos a demostrar las otras dos propiedades de las relaciones de orden (tr. $6 \ln .40)$.

Con las desiciones tomadas Ángela ha cambiado su punto de vista expresado en el diálogo inicial y las EPP integran los otros puntos de vista expresados para demostrar la conjetura, concientes de no haber resuelto el problema en términos del conjunto $A$ y de sus elementos.

En relación con la conformación del argumento. Por una parte, en el texto de Loli cada renglón, conformado por las columnas Afirmación, Razón, muestra procedimientos algebraicos correctos, aunque las razones que permiten los pasos de un renglón a otro no siempre son congruentes; sin embargo, tienen una función argumentativa dentro de la demostración que están empleando, es decir, tienen un valor epistémico. Por otra parte, el que la proposición ( $\mathrm{si} \mathrm{a}<\mathrm{b}$ entonces existe $\mathrm{c}>0$ tal que $\mathrm{a}+\mathrm{c}=\mathrm{b}$ ) haya sido tratada como una frase descompuesta en los segmentos ( $\mathrm{si} \mathrm{a}<\mathrm{b}$ entonces existe $\mathrm{c}>0)$ y $(\mathrm{a}+\mathrm{c}=\mathrm{b})$ conectados entre sí por tal que (ver Figuras 3,4) permitiéndoles asumir como hipótesis (si $a<b$ entonces existe $c>0$ ) y como tesis $(a+b=c)$ puede verse como una falta de control sobre las consideraciones estructurales de la implicación.

Las EPP produjeron un texto demostrativo. Los valores lógicos de las proposiciones que llevaron desde la hipótesis a la tesis, es correcto y, asimismo, ocurre con algunos de los significados de esas proposiciones en tanto sus valores operativos, con escepción de la sustitución (renglón 4 del bloque afirmación, razón en Figura 4). Pero, los valores teóricos que permitirían comprender el texto como una demostración están ausentes, como lo revelan tanto la introducción de la proposición (si $\mathrm{a}<\mathrm{b}$ entonces $\mathrm{b}-\mathrm{a}>0$ ) como definición de la rela- ción $\mathrm{a}<\mathrm{b}$ en $\mathrm{Q}$, así como que la proposición ( $\mathrm{si} \mathrm{a}<$ $\mathrm{b}$ entonces existe $\mathrm{c}>0$ tal que $\mathrm{a}+\mathrm{c}=\mathrm{b}$ ) no es vista como otra definición de esa misma relación.

Resalta el que la corrección de los valores lógicos esté anclada a dos procedimientos típicos: despeje de ecuaciones e introducción de una definición; fallando en el caso de la sustitución. Por último, y de manera colateral, se encuentra que la idea de demostración está asociada a la configuración en formatos de dos columnas Afirmación-Razón (Figuras 2,4).

Lo documentado hasta aquí, evidencia la emergencia de una demostración caracterizable como paraformal.

El siguiente dato corresponde a la séptima sesión. Las estudiantes están convencidas a través de la experimentación que 1 y 0,9 periódico son el mismo número. Sin embargo, se han propuesto la tarea de producir una demostración formal de este hecho matemático.

A: Pero mire que aquí podemos ver, el día en que la embarramos, que Loli dijo que si a es menor que $b$ entonces, y pienso que ese entonces era algo de lo que habíamos partido que no habíamos demostrado, porque habíamos dicho que a era menor que b.

$[\ldots]$

K: Entonces hagamos lo que dice Loli, podemos hacer una demostración formal para afirmar que son iguales.

A: bueno y ¿de qué partimos? Porque no podemos decir que a y bon iguales, ¿no?

K: y luego sigue entonces.

A: por eso ¿cómo ponemos el antes del entonces?

$\mathrm{L}: \mathrm{Si}$..., no porque tendría que ser igual, si a es igual $\mathrm{a} b$, entonces... 


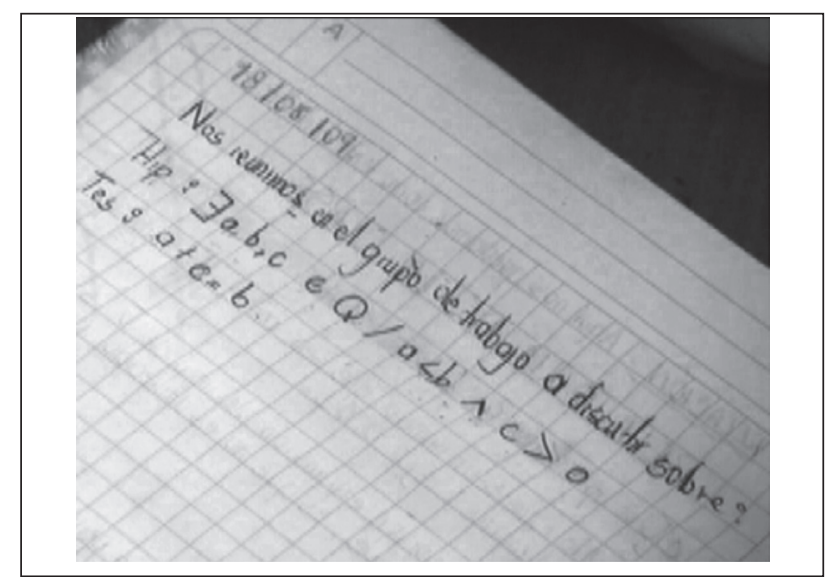

Figura 3.

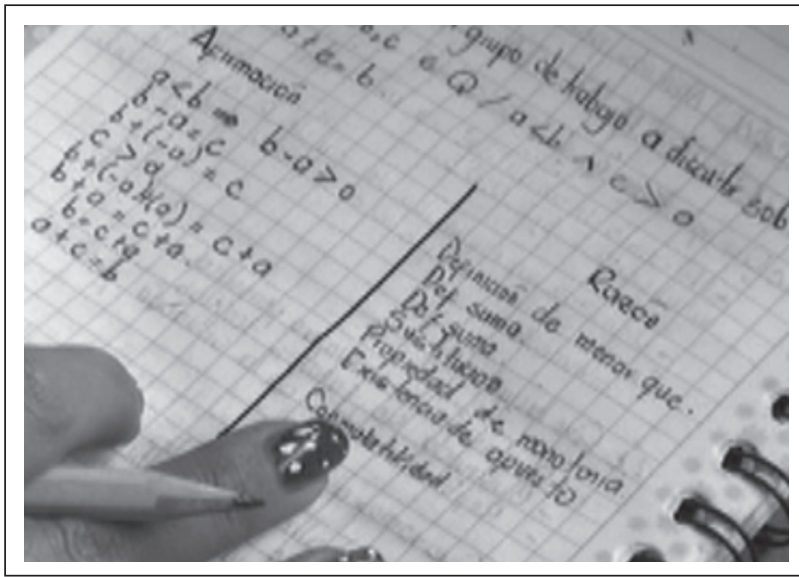

Figura 4.

K: Se quiere llegar a que ambos son iguales ¿no?

A: Si a es igual a 1, y b es igual a 1, entonces a es igual $a b$.

$[\ldots]$

L: ¿Pero cómo decimos que 1 es igual a 1? ¿Cómo demuestro eso?

A: Pues eso ya es reflexibilidad uno es igual a uno mismo.

$\mathrm{K}$ : [Asiente con la cabeza].

A: No porque mire [escribe en el puesto], si a es igual a uno y b es igual a uno, entonces a es igual a b. Entonces acá no hay que demostrar nada, porque ya tenemos eso es transitividad. Cosas iguales a una misma cosa son iguales entre sí

Transcripción 8. Reaparición de la demostración paraformal
En el intento de demostrar que 1 y 0.9 son iguales, las estudiantes recuerdan el insuceso anterior de haber hecho una demostración paraformal (tr. 6), lo que las llevó a centrarse en discernir en las proposiciones de la forma "si... entonces...», qué va antes del entonces y qué va después; para no recaer en tomar la tesis como hipótesis para el caso que se propusieron demostrar.

Aunque, como les ocurrió en un caso anterior, también tuvieron un desplazamiento desde su objetivo inicial de demostrar que igual a 1 hacia demostrar la proposición "Si $a$ igual a 1 y $b$ igual a 1 entonces $a$ igual a $b$ ", la discusión actualizada a esta proposición deja ver una argumentación en la que controlan y diferencian el papel de las hipótesis, las razones y la tesis; sin embargo, la diferencia entre lo que argumentan y el texto que el grupo propone como demostración, plasmado en el cuaderno de Katherine (Figura 5), parece una manifestación de la interferencia de la demostración paraformal.

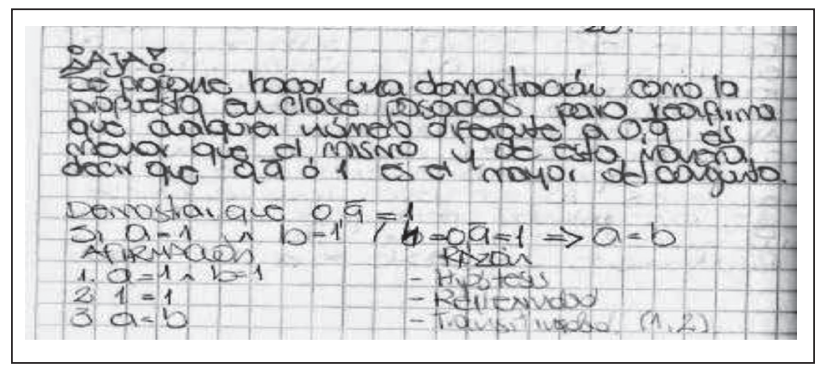

Figura 5. Reaparición de la demostración paraformal

El grupo manifiesta una conciencia de que esta clase de textos no son demostraciones en sí (ln. 5-7, tr. 8), pero le atribuyen el nombre de demostración formal (ln. 8). Realmente, se trata de una demostración paraformal porque están asumiendo la tesis como hipótesis (ln. 16, numeral 1, Figura 5) y hay una validez de cada proposición (valor lógico correcto), pero estatus teórico y operativo de las mismas no aparece (ln. 18-19, p. ej.). No obstante, expresan una conciencia de la importancia de no asumir la tesis y distinguir la hipótesis de la tesis (ln. 9-11). 
Asimismo, persisten dos ideas y dos ausencias. En primer lugar, la idea de que la demostración formal es una configuración a dos columnas de afirmaciones y razones (Figuras 4,5 ) y que en la demostración formal se deben usar proposiciones conteniendo términos de teorías, aunque no exista una relación clara con la proposición objeto de demostración -por ejemplo, de los conjuntos parcialmente ordenados- y en segundo lugar ausencias de estatus operativo y de estatus teórico de las proposiciones usadas.

En general se puede decir que mientras los estudiantes interactúan y van produciendo sincrónicamente un texto oral y un texto escrito, usando a la vez lenguaje natural y lenguaje matemático en sus argumentaciones, tienen control y diferencian el papel de las hipótesis, las razones y la tesis; su actividad matemática es completamente regulada. Pero, al situarse en la perspectiva de hacer una demostración formal, su pretensión los conduce a textos escritos con características de demostración paraformal; es decir, la suposición de la tesis como hipótesis o la introducción de una proposición equivalente a la proposición objeto de demostración. Esto sucede incluso cuando el grupo de estudiantes manifiesta, reiteradamente y de distintas maneras, que no se debe suponer para demostrar lo que se va a demostrar. En la intención de hacer una demostración formal la conciencia de tal imposibilidad no los habilita para realizar un tránsito adecuado del valor epistémico al valor de verdad.

También creen que las proposiciones tienen una validez en sí mismas. Es decir se busca que se cumpla su valor lógico, pero hay proposiciones que no tienen significado, en tanto su estatus es teórico y operativo. Este hecho puede llegar a hacer imposible, sin un tratamiento didáctico adecuado, que el grupo de estudiantes sea conciente de las relaciones funcionales y estructurales de la demostración.

\section{Conclusiones y discusión}

La participación en el experimento de enseñanza permitió la emergencia de dos sentidos primitivos de lo que significa demostrar: uno institucional y personal, y otro no institucional y ajeno.
El primero es el sentido de la demostración como una forma discursiva que permite convencerse $y$ convencer a los demás de la veracidad matemática de un resultado. Esto se realiza con procesos como conjeturar, refutar, experimentar, deducir, decantar, etc. Esta forma discursiva coincide con los significados institucionales de la matemática, en tanto se efectúan operaciones correctas y argumentos de la matemática; asimismo, coincide con el significado institucional en la comunidad de educadores matemáticos de lo que significa una demostración que explica y coincide con un sentido que Lakatos adjudica a la demostración (Lakatos, 1976).

El segundo, la demostración paraformal puede ser considerada como una forma discursiva en la que los EPP no tienen un dominio y no pueden regular el proceso de demostración. No saben con claridad a dónde quieren llegar, qué es lo que están demostrando, etc. Por otro lado, este sentido de lo que es una demostración no coinicide con los sentidos institucionales de los que significa demostrar.

Los estudios realizados anteriormente sobre demostración, Balachef (1987), Hanna (1990) y Simon (1996), concuerdan con este estudio en que la experimentación y la argumentación van de la mano. Los estudiantes usaron los ejemplos y los contrajemplos, estimulados por sus preguntas y las del profesor.

Sin embargo, por una parte, el hecho de no exponer una situación con la petición de "encuentre que" o "demuestre que", y, a cambio, exhibir una situación sobre la que los estudiantes deben inquirir, conlleva a que la experimentación sea usada para producir preguntas y conjeturas y que la conjetura principal sea un producto de conjeturaciones y refutaciones más pequeñas, articulando cilos atasco/ajá, que a su vez configura una práctica regulada que, apoyada en el cuaderno del resolutor, parece irle confiriendo sistematicidad y estrucura. Por otra parte, la configuración de la gestión de la clase en pequeños grupos colaborativos va introduciendo a los EPP en las tareas propias de una comunidad matemática -así la conformada por ellos sea una micro-comunidad- guiada por un sentido quasi-empirista de las matemáticas (Lakatos, 1976). 
Otro hecho, que difiere un poco de la teoría de la demostración que explica (Simon, 1996; Hanna, 1990; Balachef, 1987), es que la aparición de los niveles experimentales, no ocurre necesariamente de ese modo lineal. Al parecer debido a la presencia de preguntas y conjeturas, conjeturaciones y refutaciones articulando cilos atasco/ajá.

Para la teoría cultural de la objetivación (Radford, 2006), el aprendizaje consiste en dotar de sentido a los objetos que el estudiante encuentra en su cultura y a que la cultura del estudiante está inmersa en contextos institucionales (Radford, 2008). En este sentido, los estudiantes han tenido un contacto con el objeto de demostración en cursos anteriores, en donde al parecer han privilegiado prácticas como la demostración a dos columnas, el uso de los libros como condición para argumentar, etc. Estas prácticas privilegian un uso de la demostración que no tiene relación con sus características estructurales ni funcionales. Lo que nos lleva a coincidir con las afirmaciones de Hoyles (1997) y Harel (1998), citados por Perry, Camargo, Samper y Rojas (2006), cuando formulan como un obstáculo para el aprendizaje de la demostración

[...] la presentación a dos columnas, como usualmente se organizancia las demostraciones en geometría, lleva a que muchos estudiantes conciban la justificación como un ritual, en el cual la forma es más importante que el contenido [...] la imposición de un formato desvía la atención de los estudiantes hacia la forma más que hacia la coherencia argumentativa (p. 39).

Ahora bien, desde la perspectiva antropológica y sociocultural de comunidades de práctica Radford (2006), no se puede afirmar que los estudiantes no tengan un sentido de la demostración. Lo tienen, tanto de la demostración que explica, como de la demostración que demuestra.

Así, de la demostración que demuestra, tienen la idea correcta de que cada afirmación debe ser cierta, y que ellas conforman una trama conduciendo de una hipótesis a una tesis, lo que, si bien es necesario en una demostración, no es suficiente.
De la demostración que explica, en cambio, tienen bastante objetivado. No solo porque han tenido una práctica exitosa en esta dirección, sino porque además pueden hacer explícitos algunos hechos de la demostración que explica. Esto se puede ver en la siguiente intervención en el Sistema Moodle de Katherine, el 4 de octubre, a la pregunta de un foro que decía «¿Cuál es el sentido de la demostración en el curso?»

A partir de las demostraciones se puede ver la complejidad de las situaciones problema, pues por medio de éstas se ve el proceso de justificación y explicación del mismo, además sirve para no solo convencerse a uno mismo sino poder argumentar a los demás del porque se hace las cosas, su finalidad y de alguna manera refleja los conocimientos que se han ido adquiriendo, la apropiación que se tienen de estos.

Una manera de interpretar lo dicho por Katherine desde la perspectiva antropológica es con el concepto de ladera de generalidad (Radford, s. f.). La demostración en la formación de profesores puede tener, al menos, dos laderas de generalidad. Una caracterizada por la comunidad de educadores matemáticos como la demostración que explica. La otra practicada por los matemáticos y caracterizada por epistemólogos y educadores matemáticos como la demostración que demuestra.

Se puede decir, entonces, que la comunidad de aprendices ha objetivado la ladera de generalidad de la demostración que explica; sin embargo, el proceso de objetivación de la demostración que demuestra hasta ahora ha empezado; vincula tres ideas, no se puede asumir la tesis como hipótesis, se debe verificar el valor de verdad de cada proposición que constituye la demostración, y debe haber una trama argumentativa. Los valores epistémicos y los significados de los estatus teóricos y operativos de las proposiciones no están objetivados por la comunidad de aprendices.

Como se ha demostrado, (Sanjuán \& Romero, 2009; Sanjuán, Romero \& Bonilla, 2008), en concordancia con investigaciones sobre el aprendizaje de las matemáticas y en particular de la demostra- 
ción matemática, permiten deducir la complementariedad de las perspectivas asumidas, enfatizando en el hecho de que la participación en entornos de aprendizaje que vincule dichas perspectivas, permite a los EPP participar en la práctica de la demostración matemática, aprendiendo en ella aspectos que desbordan el objeto matemático, para sumergirse en el mundo de vida al involucrar maneras de hacer, particularmente matemáticas, y de ser con otros, Radford (2006).

La demostración, como objeto de la educación matemática, ha sido tratado tradicionalmente como un medio para obtener certeza (verdad); sin embargo, en la perspectiva que Villord denomina verificación/convicción, estamos de acuerdo con el autor cuando señala que aquélla no es la función más importante que se le puede adjudicar a la demostración en el contexto escolar y que más bien debe incorporarse las funciones de descubrimiento, comunicación y sistematización; todas ellas tendientes a buscar que la demostración se presente como una actividad matemática provista de sentido para los estudiantes.

En particular para los EPP que están en los procesos de aprender a enseñar, es útil participar en procesos de construcción de sentido sobre la demostración que les permita aprehender que existen diferentes sentidos para la demostración y no exclusivamente el de la demostración formal y que, la demostración puede ser vista como un proceso comunicativo propicio para construir y comprender objetos matemáticos.

\section{Agradecimientos}

Agradecemos al Centro de Investigaciones y Desarrollo Científico de la Universidad Distrital Franciso José de Caldas (CIDC-UD) por la financiación de este trabajo. Asimismo, agradecemos a los estudiantes de los cursos de Problemas del Continuo de los semestres 2008-3, 2009-1 y 2009-3 por su paciencia al permitir que su increible trabajo fuera registrado en cámaras para esta investigación.

\section{Bibliografía}

Balacheff, N. (1987). Processus de preuve et situations de validation. Eduactional Studies in Mathematics. núm. 18, pp.147-176.

Bohórquez, A. \& Sanjuán, A. (2008). Consideraciones sobre la resolución de problemas en la actualidad [en línea]. En: Memorias del IX Encuentro Colombiano de Matemática Educativa. Bogotá: Asocolme. Disponible en http:// edumat.arturosanjuan.com

Callejo, M, Valls, J., Llinares, S. (2007). Interacción y análisis de la enseñanza. Aspectos claves en la construcción del conocimiento profesional. En Investigación en la Escuela. pp. 5-21.

Cobb, P., Confrey, J., Disessa, A., Lehrer, R. \& Schauble, 1. (2003). Desing experiments in education research. En Education Researcher. Vol. 32, núm. 1, pp. 9-13.

D’Amore, B. (2006). Didáctica de la Matemática. Bogotá: Editorial Magisterio.

D’Amore, B., et. al. (2006a). El sentido del infinito. En Epsilon, Revista de la Sociedad Andaluza de Educación Matemática "Thales", núm. 65 (número especial), pp. 187-216.

Duval, R. (1999). Semiosis y pensamiento humano. Registros semióticos y aprendizajes intelectuales. Cali: Universidad del Valle.

Fischbein, E. (1990). Intuition and information processing mathematical activity. En International Journal of Science Education. Vol. 14, núm. 1, pp. 31-51.

Gavilán, J., García, M. \& Llinares, S. (2005). Una perspectiva para el análisis de la práctica del profesor de matemáticas. Implicaciones Metodológicas. En Enseñanza de las Ciencias.

Goldstern, M. \& Judah, H. (1998). The Incompleteness Phenomenon: A New Course in Mathematical Logic. Nueva York: AKPCiencias, vol. 25, núm. 2, pp. 157-170. 
Gravemeijer, K. (2004). Local instruction theories as means of support for teachers in reform mathematics education. En Mathematical Thinking and Learning, vol. 6, núm. 2, pp. 105-128.

Hanna, G. (1990). Some pedagogical aspects of proof. En Interchange, vol. 21, núm. 1, pp. 6-13.

Healy L. \& Hoyles C. (1998). Justifying and proving in school. Mathematics. Londres: Institute of Education, University of London.

Hemmi, K. (2008). Students' encounter with proof: the condition of transparency. En ZDM, vol 40, núm. 3, pp. 413-426.

Lakatos, I. (1976). Proofs and refutations. The Logic of mathematical discovery. Londres: Cambridge University Press.

Lebem. (1999). Documento de acreditación previa. Bogotá: Licenciatura en Educación Básica con Énfasis en Matemáticas, Universidad Distrital Francisco José de Caldas.

Lemke, D. (1978). Pasos hacia un cúrriculo flexible. Chile: Unesco Orelalc.

Llinares, S \& Olivero, F. (2008). Virtual communities and networks of prospective teachers. En: Krainer, Wood (Eds). Participants in Mathematics Teacher Education (pp. 155-17 ) Sense Publishers.

Mason, J., Burton, L. \& Stacy, K., (1988). Pensar matemáticamente. Editorial Labor.

Otte, M. (1994). Mathematical kKnowledge and the problem of proof. En Educational Studies in Mathematics, vol. 26, núm. 4, pp. 299-321.

Perry, P., Camargo, 1., Samper, C. \& Rojas, C. (2006). Actividad demostrativa en la formación inicial de profesores de matemáticas. Bogotá: Universidad Pedagógica Nacional.
Popper, K. (1959). The Logic of Scientific Discovery. Hutchinson \& Co.

Radford, L. (2006). Elementos de una teoría cultural de la objetivación. En Relime, (número especial), pp. 103-129.

Sanjuán, A. \& Romero, J. (2009). La toma de conciencia de la demostración en la formación de profesores: un estudio de caso. En: $\mathrm{Me}$ morias de la VII Reunión de Didáctica de las Matemáticas del Cono Sur.

Santos-Trigo, M. (2007). Mathematical problem solving: and evolving research and practice domaing. En Problem solving around the word: summing up the state of the art. ZDM Mathematics education. Vol. 35, núm. 5-6. Berlin. Springer.

Simon, M. (1996). Justification in the mathematics classroom: a study of prospective elementary teachers. En Journal of Mathematical Behavior, vol. 15, núm. 1. pp. 3-31.

Simon, M. (1996a). Beyond inductive and deductive reasoning: the search for a sense of knowing. En Educational Studies in Mathematics, vol. 30, núm. 2, pp. 197-210.

Steffe, L. \& Thompson, P. (2000). Teaching experiment methodology: Underlying principles and essential elements. En R. Lesh \& A. E. Kelly (Eds.), Research design in mathematics and science education (pp. 267-307). Hillsdale, NJ: Erlbaum.

Tall, D. (1989). The nature of mathematical proof. En Mathematics Teaching, núm. 127, pp. 28-32.

Wenger, E. (2001). Comunidades de práctica: aprendizaje, significado e identidad. Buenos Aires: Paidós. 